\title{
Dark energy: myths and reality*
}

\author{
V.N. Lukash ${ }^{\dagger}$, V.A. Rubakov ${ }^{\ddagger}$ \\ ${ }^{\dagger}$ Astro Space Centre of P.N. Lebedev Physical Institute, \\ lukash@asc.rssi.ru; \\ ${ }^{\ddagger}$ Institute for Nuclear Research, \\ rubakov@ms2.inr.ac.ru
}

October 22, 2018

\begin{abstract}
We discuss the questions related to dark energy in the Universe. We note that in spite of the effect of dark energy, large-scale structure is still being generated in the Universe and this will continue for about ten billion years. We also comment on some statements in the paper "Dark energy and universal antigravitation" by A.D. Chernin [4].
\end{abstract}

\section{Introduction}

The emergence of the idea that the entire visible Universe is permeated by a weakly interacting substance known as dark energy was the number one sensation in physics at the turn of the century and came as a complete surprise to most scientists, particularly those studying topics related both to cosmology and to particle physics. This is because the known energy scales of fundamental interactions are of the order of $1 \mathrm{GeV}$ for the strong interaction and $100 \mathrm{GeV}$ and $10^{19} \mathrm{GeV}$ for the weak and gravitational interactions, respectively. Thus, there was no reason 11 to assume that a new energy scale much smaller than the above-mentioned ones exists in nature. But it turned out that dark energy is characterized by the scal $2^{2} E_{\mathrm{V}} \sim 10^{-3} \mathrm{eV}$ defined by the relation $\rho_{\mathrm{V}}=E_{\mathrm{V}}^{4}$, where $\rho_{\mathrm{V}}$ is the dark energy density.

\footnotetext{
*Published: Physics-Uspekhi 51(3), pp 283-289 (2008).

${ }^{1}$ Apart from some arguments based on the anthropic principle, see [1-3].

${ }^{2}$ We follow the notation used in Chernin's article [4]: the subscripts V, D, and B respectively stand for dark energy, dark matter, and baryons.
} 
Moreover, in the present-day Universe, the following equality is valid within an order of magnitude:

$$
\rho_{\mathrm{V}} \approx \rho_{\mathrm{D}} \approx \rho_{\mathrm{B}}
$$

where $\rho_{\mathrm{D}}$ and $\rho_{\mathrm{B}}$ are mass densities of dark matter and baryons (protons and nuclei). And again, there is no clear a priori reasons for this equality. We point out that the approximate relation $\rho_{\mathrm{D}} \approx \rho_{\mathrm{B}}$ has been valid at each instant of cosmological evolution since the baryon asymmetry emerged and dark matter was generated, because $\rho_{\mathrm{D}}$ and $\rho_{\mathrm{B}}$ decrease with the expansion of the Universe at the same quite high rate. On the other hand, $\rho_{\mathrm{V}}$ does not depend, or barely depends, on time; hence, it is today, i.e., after the structure has appeared and stars have been formed, that the first equality in (1) is satisfied. It is certainly not easy to admit that relation (1) holds merely accidentally.

Because the properties of dark energy are very interesting and the problem itself is fundamental, it is important to understand what kind of data made scientists believe that dark energy does exist. This knowledge is necessary when we try to find explanations, which may seem exotic today, of why the expansion of the Universe is accelerating and when we choose key experiments to verify various hypotheses. We mention one such attempt below. Attempts to explain approximate relation (1) also deserve attention.

These issues are primarily considered in A.D. Chernin's paper [4]. However, we believe that that paper somewhat mythologizes dark energy because on the one hand, some crucial results are hardly mentioned there, and on the other hand, some issues discussed in that article have nothing to do with dark energy. Besides, the attempt to explain relation (1) using some 'internal symmetry in cosmology' is, to put it mildly, highly disputable.

In this paper, we try to separate the dark energy mythology from the real state of affairs.

\section{Structure argument and supernovae}

Type-Ia supernovae are often used (Chernin also writes about them) as the main observational argument confirming the existence of dark energy. But there are quite a number of other arguments, at least equally serious, based on combinations of cosmological data. Some of them were known before the observational data on type-Ia supernovae appeared, which had made several cosmologists [5-9] (see [10] for a review of the earlier papers) insist on the existence of dark energy in nature even before the first results on supernovae were available. 
One of the independent arguments is as follows. By the mid-1990s, the analysis of galactic catalogues aimed at revealing the distribution of matter in space, the use of various methods to determine the mass of clustered (clumped) matter 3 and measurements of the cosmic microwave background and the Hubble parameter had already led to the conclusion that the total mass density of nonrelativistic matter, which constitutes the inhomogeneous structure of our Universe, such as galaxies and their formations (groups, clusters, filaments, walls, superclusters, voids), is not greater than 30 percent of the critical density $\rho_{\mathrm{c}}$ :

$$
\Omega_{\mathrm{M}} \equiv \frac{\rho_{\mathrm{M}}}{\rho_{\mathrm{c}}} \leq 0.3
$$

where

$$
\rho_{\mathrm{M}}=\rho_{\mathrm{D}}+\rho_{\mathrm{B}}, \quad \rho_{\mathrm{c}}=\frac{3 H_{0}^{2}}{8 \pi G} \simeq 10^{-29} \mathrm{~g} \mathrm{~cm}^{-3},
$$

and $H_{0} \simeq 70 \mathrm{~km} \mathrm{~s}^{-1} \mathrm{Mpc}^{-1}$ is the Hubble parameter. Result (2) is one of the most important facts in modern cosmology. For a long time, it has been interpreted as evidence of the Universe having nonzero curvature. Indeed, if dark energy is not taken into account, then the Friedmann equation for the open cosmological model, written for the present epoch, reduces to the relation

$$
\rho_{\mathrm{c}}=\rho_{\mathrm{M}}+\frac{3}{8 \pi G R_{\kappa}^{2}},
$$

where $R_{\kappa}$ is the present curvature radius of space. According to (2), the curvature (the second term in the right-hand side) dominates, giving not less than $0.7 \rho_{\mathrm{c}}$.

But this interpretation faced difficulties. First, from the theoretical standpoint, a distinctly nonzero spatial curvature is almost incompatible with the inflationary Universe paradigm because inflationary models without fine tuning result in extremely small values of the spatial curvature $R_{\kappa}^{-2}$. Second, the present age of the Universe in the open model is around 11 billion years, whereas estimations of the age of the oldest objects in the Universe (for example, globular clusters) have yielded greater values of 12 to 14 billion years. There were also other arguments against the open model with a large spatial curvature.

If the spatial curvature is zero, result (2) suggests that not less than $70 \%$ of the energy density in the modern Universe is due to matter of a

\footnotetext{
${ }^{3}$ Measurements of peculiar velocities of galaxies in clusters and superclusters, gravitational lensing by clusters, measurements of galaxies' rotation curves, determination of the mass-luminosity relation, measurements of X-ray clusters' temperatures, etc.
} 
type that cannot be perturbed by gravitational fields of the structures and remains unclumped (unclustered) in the course of cosmological evolution. This implies that the effective pressure of the matter is negative 4 and its absolute value is sufficiently large, i.e., $p \approx-\rho$. Hence, this is dark energy.

The model with spatial curvature was finally discarded based on measurements of the cosmic microwave background anisotropy, or, to be more precise, the determination of the first peak position in the angular spectrum of the anisotropy, this peak being most sensitive to the value of the spatial curvature. Thanks to these measurements, it was already clear in 19992000 that the three-dimensional space is Euclidian to a high precision (i.e., $R_{\kappa}^{-1}$ is close to zero). Here, a key role was played by the balloon experiments BOOMERANG (Balloon Observations of Millimetric Extragalactic Radiation and Geophysics) and MAXIMA (Millimeter Wave Anisotropy Experiment Imaging Array) [11-14]. Later, the WMAP (Wilkinson Microwave Anisotropy Probe) experiment and others confirmed this result. Thus, the total energy density of all sorts of matter must indeed coincide with the critical density and, hence, dark energy does exist in nature.

The structure argument based on the measurements of the microwave radiation anisotropy and polarization, combined with data on the large-scale structure of the Universe, is presently a clear evidence for the existence of dark matter. We also note the integrated Sachs-Wolfe effect, which has recently been confirmed in observations. In the future, this effect should become one of the most precise methods to measure the properties of dark energy [15].

To illustrate the importance of the combination of all cosmological data on dark energy, we mention, for example, an attempt to explain the typeIa supernova observational data alternatively (see review [16] and a recent discussion in [17]) based on an assumption that matter density in our part of the Universe is significantly lower than the average value. Analysis shows [17] that this model may be consistent not only with the supernova data but also with those on microwave radiation. But whether the model will fit the results on large-scale structure and other cosmological data is highly questionable.

\footnotetext{
${ }^{4}$ We note that the condition of dominance over nonrelativistic matter excludes relativistic particles because their energy density decreases at a higher rate than the nonrelativistic matter density.
} 


\section{Hubble flows and their distortion}

Among the independent arguments supporting the existence of dark energy, Chernin considers the measurements of local cold flow (the author uses the term 'Hubble flow,' which is not quite accurate), which he discusses in great detail. Unfortunately, here we face a mythologization of dark energy.

The main theses in paper [4] and some previous articles by Chernin et al. are based on the statement that Hubble's law manifests itself even at cosmologically small distances, which is explained by antigravitation. For example, on pages 278 and 279, we read, "...antigravitation is actually capable of driving galaxies' motion almost in the entire range of cosmological distances, both at the global, 'genuinely' cosmological scales and at scales of just a few megaparsecs," "...antigravitation also dominates in our nearest galactic environment at the distance of just 1-2 Mpc from the Milky Way," "...it is the dark energy... that actually lies behind Hubble's discovery and makes sense of it for cosmology," "...dark energy can be... measured in every place where a regular outflaw of galaxies is observed." But we show in this section that, as a matter of fact, dark energy has not yet influenced the local velocity distribution to the full extent, and the expansion in accordance with the Hubble law starting from the scale of several megaparsecs is excluded. What does define the local flow properties is the profile of the spatial density perturbation spectrum.

The initial Hubble flows existed throughout the entire Universe. The flows in different regions were destroyed at different times, in a manner that directly depended on the forming structure.

As is known, the structure of the Universe has resulted from gravitational amplification of density perturbations whose initial amplitudes were about $10^{-5}$ for wavelengths equal to the Hubble size at that time. The perturbations were growing faster for short waves. As a result, the nonlinearity scale at which the Hubble flows are completely destroyed (the dark matter and baryon perturbations $\delta_{\mathrm{M}} \equiv \delta \rho_{\mathrm{M}} / \rho_{\mathrm{M}} \sim 1$ ), was increasing with time. In the present-day Universe, the average value of this scale is around $15 \mathrm{Mpc}$, varying, however, between different regions of the Universe. For example, it is smaller at a far distance from galaxy clusters (which are the most massive gravitationally bound formations). In particular, the nonlinearity scale in our local region is around $2 \mathrm{Mpc}$ (the size of the Local Group of galaxies).

In quasilinear regions, where the density perturbations are still not high $\left(\delta_{\mathrm{M}}<1\right)$, galaxies continue outflowing in accordance with the initial conditions. But the Hubble flows in such regions are also distorted. In the future, in dozens of billions of years, peculiar velocities will fade out because of the dynamic influence of dark energy, and the motion of galaxies will obey the 
Hubble law5 again, as in the early Universe.

This is our main disagreement with the thesis in [4]. Chernin believes that outside the gravitationally bound regions peculiar velocities of the galaxies have faded out owing to the dynamic influence of dark energy, and the motion obeys the Hubble law. In this section, we show that such recovery of the Hubble flows is only possible in the distant future (if dark energy has vacuum properties), as opposed to today, when the Universe is experiencing peculiar velocities that have maximum values over its history and are caused by inhomogeneities of matter density.

\subsection{Inhomogeneous Universe}

At the quasilinear stage, our Universe is described [18] by the generalized Friedmann equation 6

$$
\left(\frac{\dot{b}}{H_{\mathrm{V}}}\right)^{2}=\frac{c}{b}+b^{2}-\kappa \equiv f^{2}(b)-\kappa(\mathbf{x}),
$$

where $(t, \mathbf{x})$ are Lagrangian coordinates comoving with matter (the matter 4 -velocity is $\left.u_{\alpha}=t_{, \alpha}\right), b=b(t, \mathbf{x})$ is the volume expansion scale factor the comoving matter density is equal to $\left.\rho_{\mathrm{M}}=3 c H_{\mathrm{V}}^{2} /\left(8 \pi G b^{3}\right)\right], H_{\mathrm{V}}=H_{0} \sqrt{\Omega_{\mathrm{V}}} \simeq$ $2 \times 10^{-4} \mathrm{Mpc}^{-1}$ is the Hubble parameter of dark energy, and

$$
c \equiv \frac{\Omega_{\mathrm{M}}}{\Omega_{\mathrm{V}}}=\frac{\Omega_{\mathrm{M}}}{1-\Omega_{\mathrm{M}}} \simeq 0.39 .
$$

The function

$$
f(b) \equiv\left(\frac{c}{b}+b^{2}\right)^{1 / 2} \geq 1
$$

has a minimum $f_{\min } \simeq 1$ at $b_{\min }^{-1} \simeq 1.7$.

An arbitrary small function $\kappa=\kappa(\mathbf{x})$ of spatial coordinates describes the local spatial curvature. We are interested in the spatial regions where the right-hand side 7 of $\operatorname{Eqn}(3)$ is positive:

$$
\kappa(\mathbf{x})<1 .
$$

\footnotetext{
${ }^{5}$ Here, we are talking only about galaxies that are located in the quasilinear regions of space $\left(\delta_{\mathrm{M}}<1\right)$. In gravitationally bound systems $\left(\delta_{\mathrm{M}}>1\right)$, galaxies held by the gravitational field of these formations do not experience outflow.

${ }^{6}$ At this stage, we neglect the radiation density. The first two terms in the right-hand side of (3) respectively describe nonrelativistic matter (dark matter and baryons) and dark energy modeled by the cosmological constant. The dot over a variable denotes the partial derivative with respect to time $t$. The modern value of the cosmological scale factor is set to $a_{0}=1$ [see $\left.(5)\right]$.

${ }^{7}$ Condition (4) includes both superclusters (the regions where $\kappa>0$ ) and cosmological voids $(\kappa<0)$.
} 
If this condition is satisfied, the matter density decreases with time monotonically.

When $\kappa=0$, the volume and background scale factors are equal (although the expansion anisotropy remains large; see Section 3.2),

$$
b=a(t) \equiv \frac{1}{1+z}, \quad H \equiv \frac{\dot{a}}{a}=H_{\mathrm{V}} \frac{f(a)}{a},
$$

where $f=f(a)$ is the growth rate factor of the Hubble velocity $\left(\mathbf{V}_{\mathrm{H}}=\right.$ $\left.f H_{\mathrm{V}} \mathbf{x}\right)$.

In the general case, in the linear order in $\kappa$, we obtain

$$
\begin{gathered}
b=a\left(1-\frac{1}{3} g \kappa\right), \quad \delta_{\mathrm{M}}=g \kappa, \\
H_{\text {eff }} \equiv \frac{\dot{b}}{b}=H\left(1-\frac{1}{3} h \kappa\right), \quad h \equiv \frac{v}{f}=\frac{\dot{g}}{H},
\end{gathered}
$$

where $\delta_{\mathrm{M}} \equiv \delta \rho_{\mathrm{M}} / \rho_{\mathrm{M}}$ is the comoving density perturbation, $H_{\mathrm{eff}}=H_{\mathrm{eff}}(t, \mathbf{x})$ is the effective Hubble function, and $g=g(a)$ and $v=v(a)$ are the respective growth factors of the density perturbations and the matter peculiar velocity [see also (11)],

$$
g(a)=\frac{1}{c}\left(a-H \int_{0}^{a} \frac{\mathrm{d} a}{H}\right), \quad v(a)=\frac{3 H_{\mathrm{V}}}{2 a^{2}} \int_{0}^{a} \frac{\mathrm{d} a}{H} .
$$

Equations (3)-(8) describe quasi-Hubble flows with the effective Hubble parameter $H_{\text {eff }}$ depending on the observer's location. Figure 1 shows the functions $g(a)$ and $v(a)$. In the present era, the function $v$ is in its wide maximum, indicating the period of the most intensive structure formation. The position of the maximum of $v(a)$ corresponds to $z \simeq 0.2$, the level of 90 percent of the maximum value is reached at $a \simeq 0.5$ and 1.4 , and the half-maximum is at $a \simeq 0.1$ and 4 . Therefore, the present era is an era of maximum peculiar velocities, and it will continue to last for a cosmological time. The function $v$ will have decreased to only half its current value by the time the Universe is 35 billion years old. And only then will it be possible to talk about the era of faded-out peculiar velocities in every space region where $\kappa<1$.

Figure 2 displays the function $h=h(a)$ describing the deviation of the local Hubble parameter from the background one. The function has its maximum at $z \simeq 0.4$, and the interval $h>0.5 h_{\max }$ spans the range $a \in(0.1,1.8)$, which corresponds to the age 0.6 to 22 billion years. We can learn from Fig. 


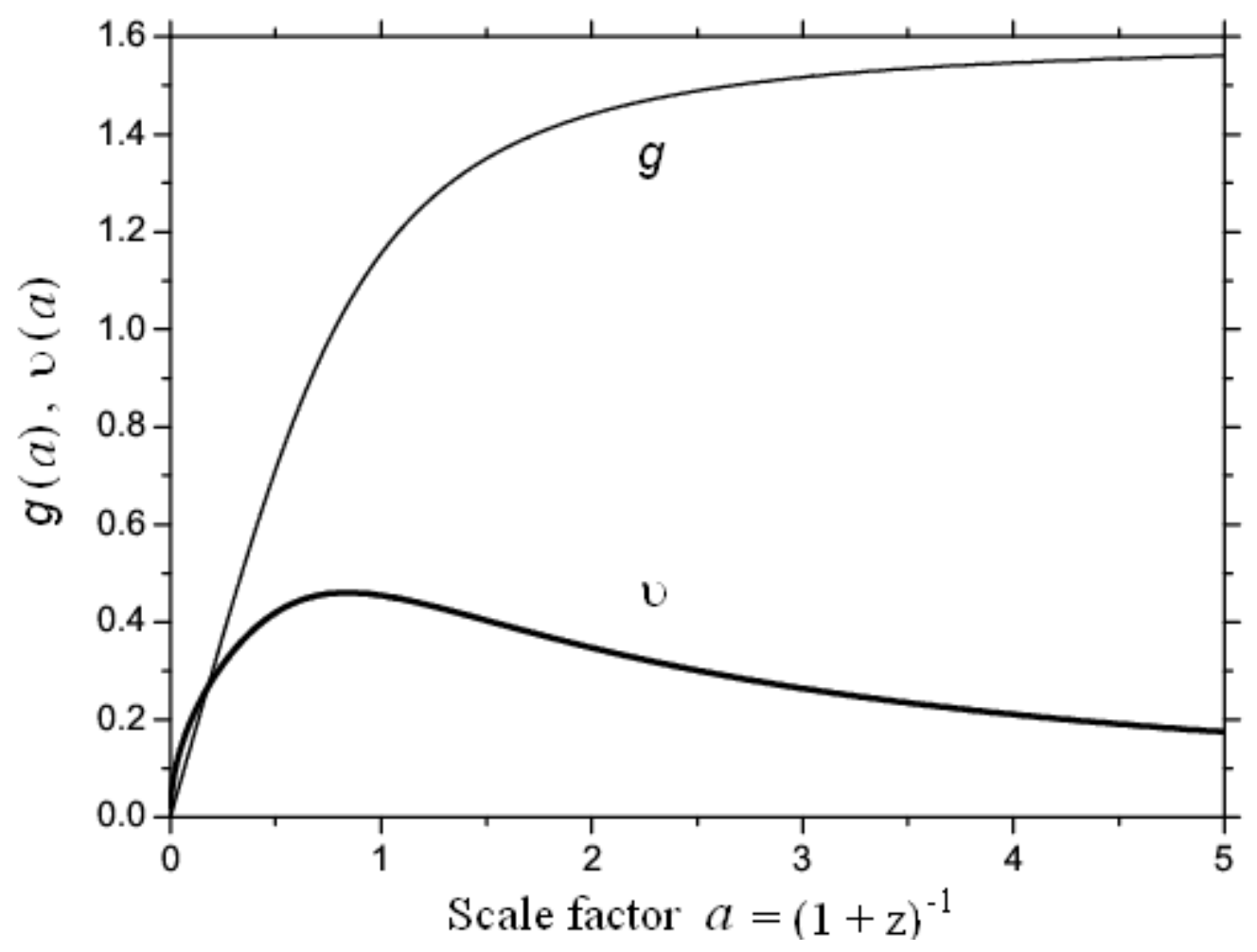

Figure 1: The functions of the density perturbation growth rate $g(a)$ and of the matter peculiar velocity $v(a)$.

2 that our Universe is at the stage of maximum distortion of Hubble's expansion, and the Hubble flows are to be recovered only in some 10 billion years.

To summarize, we can conclude that the large-scale structure formation in the Universe occurs during the period that spreads from 1 to 20 billion years since the Big Bang. The stage of the suppression of Hubble flow inhomogeneities due to dark energy's gravitational influence has not come yet. That is why one of the key theses in [4, p. 279] — that 'the dynamic effect of dark energy naturally explains the two astronomical facts that have seemed mysterious up to now: (1) regularity of the expansion flow inside the uniformity cell and (2) the same expansion rate at local and global scales' - is incorrect.

We now consider local matter flows and their properties in more detail. 


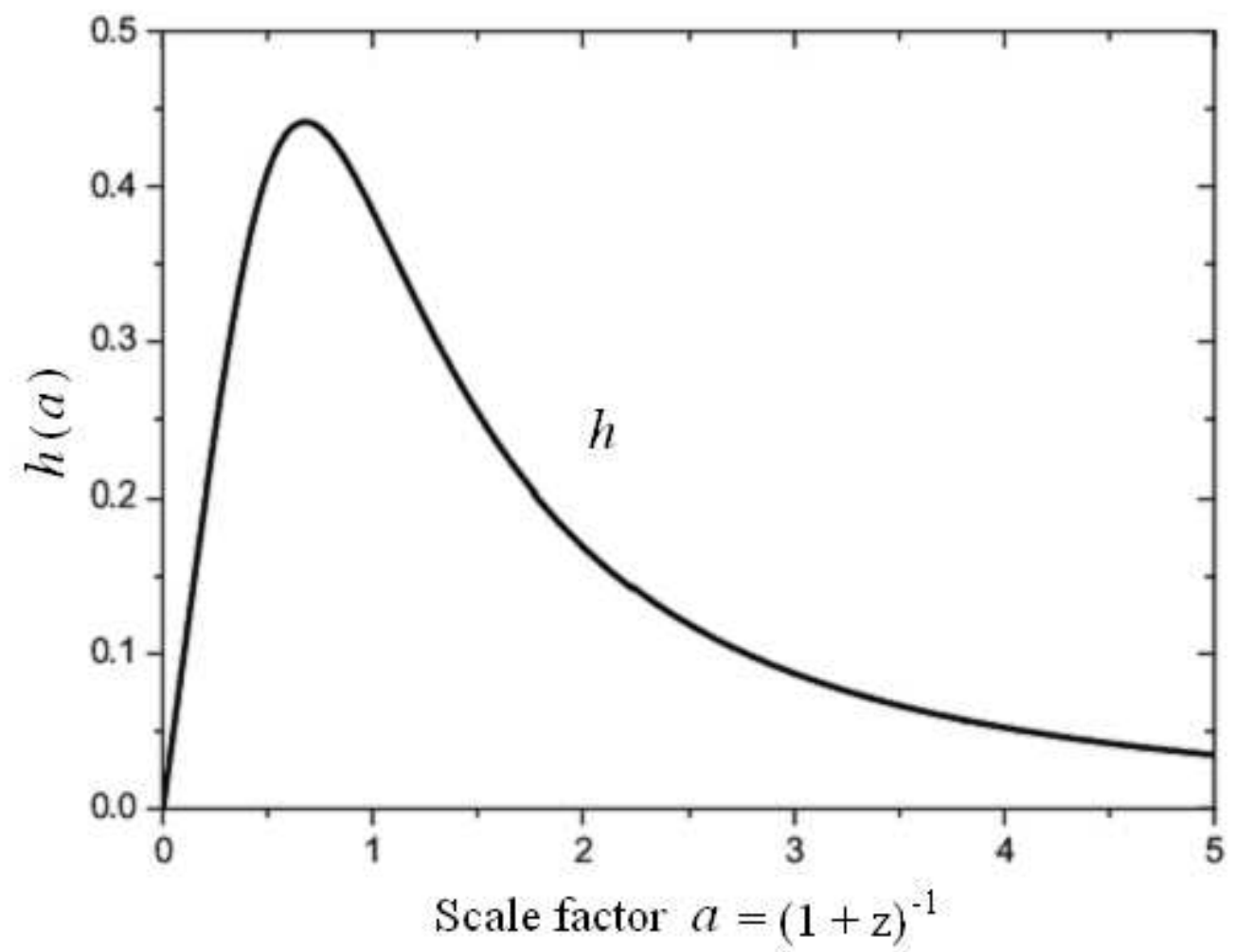

Figure 2: The function $h(a)$ describing the deviation of the local Hubble parameter $H_{\text {eff }}$ from the background one.

\subsection{Anisotropy of cold flows}

To describe the gauge-invariant field of peculiar velocities, we pass to Euler (quasi-Friedmannian) coordinates $y^{\alpha}=(\tau, \mathbf{y})$. In these coordinates, the gravitational field is locally isotropic at any spatial point in the linear order in $\kappa$. The required transformation is given by

$$
\begin{gathered}
\mathbf{y}=\mathbf{x}+g \mathbf{S}, \quad \tau=t-a v H_{\mathrm{V}} \bar{q}, \\
S_{i}=-\bar{q}_{, i}, \quad q=\frac{3}{2} H_{\mathrm{V}}^{2} \bar{q},
\end{gathered}
$$

where $\mathbf{S}=\mathbf{S}(\mathbf{x})$ is the vector of a medium element displacement from the unperturbed position 8 and $q=q(\mathbf{x})$ is a time-independent dimensionless

\footnotetext{
${ }^{8}$ We recall that the $\mathbf{x}$ coordinate does not change with time along the medium element trajectory and tends to $\mathbf{y}$ as $t \rightarrow 0$.
} 
displacement potential [18]. Comparison with (6) yields

$$
\kappa=-\operatorname{div} \mathbf{S}=\Delta \bar{q} .
$$

The matter particle displacement from the unperturbed Hubble trajectories is monotonically increasing with time and today amounts to the value $\sigma_{S} \simeq 15$ Mpc. If dark energy has vacuum properties, the displacement will tend to its asymptotic value of about $25 \mathrm{Mpc}$ in the future.

Transformation (9) yields the following form of the metric interval in the quasi-Friedmanninian and Lagrangian frames:

$$
\begin{aligned}
& \mathrm{d} s^{2}=(1+2 \Phi) \mathrm{d} \tau^{2}-\tilde{a}^{2} \mathrm{~d} \mathbf{y}^{2} \\
= & \mathrm{d} t^{2}-\tilde{a}^{2}\left(\delta_{i k}-2 g \bar{q}_{, i k}\right) \mathrm{d} x^{i} \mathrm{~d} x^{k},
\end{aligned}
$$

where $\tilde{a} \equiv a(t)(1-q)$ is a 4 -scalar invariant under coordinate transformations and $\Phi=(\mathrm{cg} / \mathrm{a}) q$ is the gravitational potential of density perturbations. The function $b(t, \mathbf{x})$ is directly proportional to the trace of the spatial part of the Lagrangian metric tensor [compare (10) and (6)].

Equation (10) gives the physical Euler coordinate of the medium element, $\mathbf{r}=\tilde{a} \mathbf{y}$. Differentiating $\mathbf{r}$ with respect to the proper time yields the following expression for the matter peculiar velocity:

$$
\mathbf{v} \equiv \dot{\mathbf{r}}-H \mathbf{r}=v H_{\mathrm{V}} \mathbf{S}
$$

Expression (11) coincides with the definition of the 3-velocity as the spatial component of the matter 4-velocity in the quasi-Friedmannian reference frame:

$$
v_{i}=-\frac{\partial t}{a \partial y^{i}}=-v H_{\mathrm{V}} \bar{q}_{, i} .
$$

Therefore, the value $v$ appearing in (7) is indeed the peculiar velocity growth rate.

We now consider the local Hubble flows. In regions (4) of the inhomogeneous Universe, the Hubble flows are described by the tensor field $H_{i k}=H_{i k}(t, \mathbf{x})$ generalizing the function $H(t)$ in the Friedmann model [see (13)]. At a fixed instant of time $t$, Eqns (9) give the coordinate distance between two close medium points:

$$
\delta y_{i}=\left(\delta_{i k}-g \bar{q}_{, i k}\right) \delta x^{k} .
$$

Differentiating the physical distance $\delta \mathbf{r}=\tilde{a} \delta \mathbf{y}$ with respect to time yields the field of paired velocities:

$$
\delta V_{i} \equiv \frac{\partial}{\partial t}\left(\delta r_{i}\right)=H_{i k} \delta r^{k}
$$




$$
H_{i k}=H \delta_{i k}-\dot{g} \bar{q}_{, i k}=H\left(\delta_{i k}-h \bar{q}_{, i k}\right) .
$$

We can see that the trace of (14) corresponds to the volume Hubble parameter $H_{\text {eff }}=(1 / 3) H_{i i}$, but the tensor $H_{i k}$ itself is highly anisotropic. The local expansion anisotropy (variations in the projections of $H_{i k}$ on directions radiating from a given point $\mathbf{x}$ ) is of the same order as the deviations of $H_{\text {eff }}$ from the true Hubble parameter $H$. For example, in the Local Group, at a distance more than $2 \mathrm{Mpc}$ from its barycenter, the expected anisotropy of the quasi-Hubble outflaw of galaxies can amount to $30 \%$.

The field $H_{i k}$ describes regular (cold) matter flows. It is worth saying that formula (13) is valid in the limit of small distances between galaxies, i.e., distances smaller than the correlation scale of the two-point correlation function of the displacement vector. The correlation radius varies from 15 to $40 \mathrm{Mpc}$ between projections of this vector with respect to the direction $\delta \mathbf{y}$. As the distance increases, random deviations from law (13) increase. This is due to the cosmological velocity perturbation spectrum, whose amplitude decreases with a decrease in the wavelength for $k>0.03 \mathrm{Mpc}^{-1}$, and hence the random deviations from average velocities (13) increase as the wavelength $k^{-1}$ increases. Just to give an example, at the distance $3 \mathrm{Mpc}$ from the Local Group barycenter, the deviations are around $30-40 \mathrm{~km} \mathrm{~s}^{-1}$, which is about $15-20 \%$ of the average velocity, while the full peculiar velocity of the Local Group relative to the microwave background is $600 \mathrm{~km} \mathrm{~s}^{-1}$. The main inhomogeneity scales responsible for such a high velocity are in the range 15 - $50 \mathrm{Mpc}$.

We can see that the standard theory of the formation of the structure of the Universe faces no 'mysterious' problems in explaining the observed relative motion of matter in quasi-homogeneous regions of the Universe $(\kappa<1)$. The local flows are regular, smooth, and highly correlated. The smallness of the random deviations in galaxy velocities from average cold flows is explained with a profile of the initial spatial density perturbation spectrum, contrarily to Chernin's evolutionary influence of dark energy. At small distances, the flows are quasi-Hubble (they are radial, the outflaw velocity being in direct proportion to distance), but the Hubble parameter depends on direction and on the observer's location. There is no reason to modify the standard theory. The 'Little Bang' mode 9 proposed in Section 3 in [4] in order to explain the cold flows is beneath criticism. In that model, the peculiar velocities of galaxies 'kicked' out of the Local Group must decrease as

\footnotetext{
9'The local cosmology' in Section 3.4 in [4], which underlies the 'Little Bang' model, is based on the limit of the static gravitational field (see Eqns. (28)-(36) in [4]). This contradicts the standard theory, where the gravitational potential $\Phi(t, \mathbf{x})$ depends on time [see Eqn. (10)].
} 
much as five times under the gravitational influence of dark energy. As Fig. 1 shows, this will take more than 40 billion years.

\section{On 'internal symmetry in cosmology'}

Chernin's suggestion to link relation (1) with mythological 'internal symmetry in cosmology' ([4], Section 5) causes serious disagreement. In this respect, the crucial point for the author is his introducing 'Friedmann integrals.' To determine their values, the author arbitrarily normalizes the scale factor (the value of the parameter $R_{0}$ in Eqn. (57) in [4]) to the present horizon size $R_{0}=H_{0}^{-1}$. With the parameter $R_{0}$ chosen differently, the equality of the 'Friedmann integrals,' e.g., those for dark matter and dark energy, $A_{\mathrm{D}}$ and $A_{\mathrm{V}}$, would be violated. It is clear that if $R_{0}=H_{0}^{-1}$, then the approximate relations between the 'Friedmann integrals'

$$
A_{\mathrm{B}} \sim A_{\mathrm{D}} \sim A_{\mathrm{R}} \sim A_{\mathrm{V}}
$$

are equivalent to the approximate relations

$$
\Omega_{\mathrm{B}} \sim \Omega_{\mathrm{D}} \sim \sqrt{\Omega_{\mathrm{R}}} \sim \frac{1}{\sqrt{\Omega_{\mathrm{V}}}}
$$

for the present ratios of the energy densities to the critical density. To verify this, it is sufficient to use the Friedmann equation for the spatially flat or almost flat Universe at today's instant, which gives

$$
A_{\lambda}=R_{0}\left[\Omega_{\lambda}\left(H_{0} R_{0}\right)^{2}\right]^{1 /\left(1+3 w_{\lambda}\right)},
$$

for all sorts of matter $\lambda$, where $w_{\lambda}=p_{\lambda} / \rho_{\lambda}$ (cf. Eqn. (59) in [4]). This last formula implies the equivalence of relations (15) and (16) within an order of magnitude. Because $\Omega_{\mathrm{B}}, \Omega_{\mathrm{D}}$, and $\Omega_{\mathrm{V}}$ are presently quite close to unity [however, see (17) and (18)], relation (16), in turn, is equivalent to the approximate equality in (1) complemented with the same relation for photons.

Thus, what the author thinks is a 'symmetry' is, in fact, the rephrased statement about the densities of different energy components being close to each other. Introducing the 'Friedmann integrals' does not make relation (1) any clearer.

The author's approach to the flatness problem ('Dicke's problem') is equally awkward. Without an extremely fine tuning of the cosmological evolution initial data at the hot stage (the tuning noted by Dicke, which is automatically fulfilled in the inflationary theory), it is impossible to obtain 
small spatial curvature in the present Universe. In the author's terms, this means that, had it not been the fine-tuning of the initial data, the dimensionless parameter in Eqn. (87) in [4] would have been extremely high, and for the closed model, the equality between the energy densities of matter and dark energy would have never been satisfied. For example, for the closed Universe and the fixed $\rho_{\mathrm{V}} \sim 10^{-29} \mathrm{~g} \mathrm{~cm}^{-3}$, it is absolutely clear that if the curvature had contributed, e.g., $10^{-6}$ of the matter contribution to the Friedmann equation in the nucleosynthesis period, the expansion of the Universe would have changed to contraction and subsequent re-collapse long before dark energy would play a role in the cosmological expansion.

In fact, relations like (1) or (16) involve some very interesting, but still unclear issues. For example, the relation $\rho_{\mathrm{D}} \approx \rho_{\mathrm{B}}$ being constant in time possibly indicates the common origin of dark matter and the baryonic asymmetry of the Universe. In spite of numerous attempts to account for this fact, no satisfactory theoretical models have been proposed. The relation $\rho_{\mathrm{V}} \approx \rho_{\mathrm{D}}$ is valid today but includes quantities changing with time at different rates; this suggests that the period of transition from the matter-dominated to the dark-energy-dominated stage occupies a privileged position on the time axis in terms of the structure (and hence life) formation.

The fact of the existence of the large-scale structure is crucial from the perspective of the coincidence problem. The relations between the parameters $\Omega_{\mathrm{R}}, \Omega_{\mathrm{V}}$, and $\Omega_{\mathrm{M}}=\Omega_{\mathrm{D}}+\Omega_{\mathrm{B}}$,

$$
\Omega_{\mathrm{R}} \ll \Omega_{\mathrm{M}}, \quad \Omega_{\mathrm{V}} \sim \Omega_{\mathrm{M}},
$$

have a direct impact on the possibility of the generation of the structure of the Universe because gravitational instability is not realized at the radiationand dark-energy-dominated stages and develops only if nonrelativistic matter dominates. But for the structure to be formed, one more condition must be satisfied: the initial perturbation amplitude must be just right to fit the 'window' of gravitational instability, thus giving rise to inhomogeneities. In our Universe, the two necessary conditions are satisfied: the initial perturbations (of the order $10^{-5}$ ) manage to grow and form the large-scale structure of the Universe during the time 'window' from 0.3 to 20 billion years. The condition

$$
\Omega_{\mathrm{R}} \ll \Omega_{\mathrm{B}}<\Omega_{\mathrm{D}}
$$

in turn, is necessary for forming stars in nonlinear halos of dark matter.

Any detailed discussion of these and similar issues would require a new review (in this respect, see, e.g., [1, 3, 19-21]). Here, we find it important to emphasize that the outlined range of issues is much richer than it may seem after reading Chernin's article. 


\section{On the physical nature of dark energy}

Almost everywhere in [4], Chernin identifies dark energy with the vacuum energy, while other possibilities are mentioned just briefly. We want to point out that a no less attractive point of view is to relate dark energy to a new superweak and superlight field, which can be a quintessence, a phantom field, etc.

It is appealing because, among other things, it is very hard to explain the vacuum energy value, which is nonzero and still extremely small compared to the energy scales of the known interactions (see Section 1). It is much easier to imagine the vacuum energy relaxing practically to zero at some stage of the evolution of the Universe (long before the known stages). There are examples of such mechanisms in the literature [22, 23]. In this framework, it is natural for dark energy to be the energy of a new field rather than the vacuum energy. Another heuristic argument is that the present stage of the accelerated expansion of the Universe looks qualitatively similar to the inflation stage and differs from the latter 'only' in the energy density value and, hence, the Hubble parameter. Dark energy in the form of a superweak field could be a less energetic counterpart of the inflaton, a field commonly used in inflationary theories.

If dark energy is the energy of a new field, the parameter $w_{\mathrm{V}}$ that relates pressure and energy density in accordance with the equation $p_{\mathrm{V}}=w_{\mathrm{V}} \rho_{\mathrm{V}}$, differs from -1 (by the way, it does not have to be constant in time), and the dark energy density depends on time. However, we emphasize that in most models of this type, the parameter $w_{\mathrm{V}}$ is automatically close to the vacuum value $w=-1$, and hence the observational limit $\left|w_{\mathrm{V}}+1\right|<0.1$ hardly constrains the existing models yet.

Finally, we note that the accelerated expansion may be caused by gravitation theory modified at superlarge scales and cosmological times. One of the possibilities is here related to the extra spatial dimensions of infinite size (for example, see Ref. [24]), although attempts to construct such models have experienced internal contradictions so far. Another possibility, more realistic from the standpoint of theoretical realization, is the extension of General Relativity to the scalar-tensor theory of gravity $[25,26]$.

Thus, the Universe's accelerated expansion may be the first evidence of new physical phenomena occurring at cosmological and maybe other scales. Various models of the accelerated expansion differ in the dark energy density dependence on time. The search for this dependence and its detailed study are important problems of observational cosmology, which must eventually allow revealing the physical nature of dark energy. 


\section{Additional comments}

Chernin's article ought to be read with caution. For example, not all researchers are that enthusiastic about the extravagant model by Luminet et al.; peculiarities, if any, in the angular spectrum of the cosmic microwave background can be explained in a less exotic way. Almost the same applies to the model by Arkani-Hamed, Dimopoulos, and Dvali (known as the ADD model). This model definitely played a great role in presenting the idea that extra spatial dimensions can be large (or even infinitely large), but it is unlikely that nature follows this way. We note further that Chernin's paper is replete with terms that are not commonly accepted, such as EGvacuum, Q-vacuum, and Friedmann integrals.

To summarize, we recommend that the concerned reader form a reasoned opinion on issues mentioned in Chernin's article using alternative reviews on this topic, e.g., Refs. [20, 27-30].

\section{Conclusion}

The discovery of dark energy dotted the $i$ 's and crossed the $t$ 's in observational cosmology. The standard cosmological model $(\Lambda C D M)$ fitting the whole set of observational data arose for the first time in the development of science. Nowadays, it has no serious rivals. The standard model describes both the evolution of the Universe as a whole and the generation of its structure remarkably well. In spite of the influence of dark energy, the structure is still being generated and this will continue for another ten billion years or so.

At the same time, with dark energy having been recognized to exist, the situation in physics has dramatically changed and we see our knowledge of the microworld as incomplete. It is a safe bet to say that revealing the physical nature of dark energy is the central problem of natural science.

\section{Acknowledgments}

The authors are grateful to A.G. Doroshkevich, M.B. Libanov, E.V. Mikheeva, and V.N. Strokov for the useful discussions. The work was supported in part by the Russian Foundation for Basic Research, Grants 07-02-00886 and 0802-00473. 


\section{References}

[1] Weinberg S., Rev. Mod. Phys. 61, 1 (1989).

[2] Martel H., Shapiro P. R., Weinberg S., ApJ 492, 29 (1998); astro-ph/9701099.

[3] Linde A., In Science and Ultimate Reality: Quantum Theory, Cosmological and Complexity (Eds J.D. Barrow et al.) (Cambridge: Cambridge Univ. Press, 2004) p. 426; hep-th/0211048.

[4] Chernin A. D., Usp. Fiz. Nauk 178, 267 (2008) [Phys. Usp. 51 (3), 267 (2008)].

[5] Kofman L. A., Gnedin N. Y., Bahcall N. A., ApJ 413, 1 (1993).

[6] Ostriker J. P., Steinhardt P. J., Nature 377, 600 (1995).

[7] Krauss L. M., Turner M. S., Gen. Rel. Grav. 27, 1137 (1995); astro-ph/9504003.

[8] Krauss L. M., ApJ 480, 466 (1997); astro-ph/9607103; ApJ 501, 461 (1998); astro-ph/9706227.

[9] Totani T., Yoshii Y., Sato K., ApJ 483, L75 (1997); astro-ph/9705014.

[10] Carroll S. M., Press W. H., Turner E. L., Annu. Rev. Astron. Astrophys. 30, 499 (1992).

[11] Melchiorri A. et al. (Boomerang Collab.), ApJ 536, L63 (2000); astro-ph/9911445.

[12] de Bernardis P. et al. (Boomerang Collab.), Nature 404, 955 (2000); astro-ph/0004404; Lange A. E. et al. (Boomerang Collab.), Phys. Rev. D 63, 042001 (2001); astro-ph/0005004.

[13] Balbi A. et al., ApJ 545, L1 (2000); "Erratum" 558, L145 (2001); astro-ph/0005124; Hanany S. et al., ApJ 545 L5 (2000); astro-ph/0005123.

[14] Jaffe A. H. et al. (Boomerang Collab.), Phys. Rev. Lett. 86, 3475 (2001); astro-ph/0007333.

[15] Pogosian L., New Astron. Rev. 50, 932 (2006); astro-ph/0606626. 
[16] CélérierM. - N., 'The accelerated expansion of the Universe challenged by an effect of the inhomogeneities", New Adv. Phys. 1, 29 (2007); astro-ph/0702416.

[17] Alexander S. et al., "Local void vs dark energy: confrontation with WMAP and type Ia supernovae", arXiv:0712.0370.

[18] Lukash V. N., Zh. Eksp. Teor. Fiz. 79, 1601 (1980) [Sov. Phys. JETP 52, 807 (1980)]; Pis'ma Zh. Eksp. Teor. Fiz. 31, 631 (1980) [JETP Lett. 31, 596 (1980)]; astro-ph/9910009.

[19] Tegmark M., Rees M. J., ApJ 499, 526 (1998); astro-ph/9709058.

[20] Weinberg S., "The cosmological constant problems", astro-ph/0005265.

[21] Rubakov V. A., Usp. Fiz. Nauk 177, 407 (2007) [Phys. Usp. 50, 390 (2007)].

[22] Rubakov V. A., Phys. Rev. D 61, 061501 (2000); hep-ph/9911305.

[23] Steinhardt P. J., Turok N., Science 312, 1180 (2006); astro-ph/0605173.

[24] Deffayet C., Dvali G., Gabadadze G., Phys. Rev. D 65, 044023 (2002); astro-ph/0105068.

[25] Boisseau B. et al., Phys. Rev. Lett. 85, 2236 (2000); gr-qc/0001066.

[26] Gannouji R. et al., JCAP (09), 016 (2006); astro-ph/0606287.

[27] Sahni V., Starobinsky A., Int. J. Mod. Phys. D 9, 373 (2000); astro-ph/9904398.

[28] Peebles P. J. E., Ratra B., Rev. Mod. Phys. 75, 559 (2003); astro-ph/0207347.

[29] Padmanabhan T., Phys. Rep. 380, 235 (2003); hep-th/0212290.

[30] Sahni V., Starobinsky A., Int. J. Mod. Phys. D 15, 2105 (2006); astro-ph/0610026. 